\title{
CRESCIMENTO EM VASOS, DE CULTIVARES DE SOJA E DE TRIGO EM FUNÇÃO DA SATURAÇÃO DE ALUMÍNIO
}

\author{
H.A.A. MASCARENHAS ${ }^{1,2}$; R.T. TANAKA ${ }^{1,2}$ \\ 'Seção de Leguminosas - LAC, C.P. 28, CEP: 13001-970 - Campinas SP.
}

\begin{abstract}
RESUMO: Um dos problemas que a acidez do solo causa às plantas é concentrar alguns elementos químicos em nível tóxico, como por exemplo de aluminio (Al) e manganês. Visando estudar esse aspecto, fol conduzido um experimento em casa de vegetaçăo, utillzando-se amostra de subsolo, classificado como Latossolo Vermelho Amarelo fase rasa, de extrema acidez e alto teor de Al trocável. A acidez do substrato foi neutralizada com doses de hidróxido de cálcio, no intuito de reduzir a saturação de Al originalmente de 67 para 45, 30, e 15\%. As incubaçōes e os cultivos foram feitos em vasos contendo $6 \mathrm{~kg}$ daquele subsolo. Inicialmente fol testada a soja variedades Biloxi, Davis e Santa Rosa, com mediçōes de: altura das plantas, número de nós, tamanho dos internódios, número de folhas trifoliadas, produção de matéria seca da parte aérea e das raizes. Imediatamente após, o sub-solo foi cultivado com trigo variedades BH-1146 e Siete Cerros, sendo medido o comprimento dos seus sistemas radiculares. Verificou-se para a soja que não houve diferenças significativas dentro de cada variedade para as saturaçōes de Al estudadas, evidenciando que todas foram tolerantes ao Al. As raizes do trigo BH-11 46 não mostraram redução no desenvolvimento em quaisquer saturações de Al. Por outro lado, as da v. Siete Cerros mostraram menor comprimento à medida que se aumentou a saturação de Al, provando sua suscetibilidade a esse elemento.
\end{abstract}

Descritores: matéria seca, soja, saturaçăo de aluminio, tolerante

\section{TOLERANCE OF SOYBEAN AND WHEAT VARIETIES IN SUB-SOIL HIGH IN EXCHANGEABLE ALUMINUM}

ABSTRACT: A pot experiment was installed in a greenhouse using an acid sub-soll, classified as a Red Yellow Latosol, shallow phase, high in aluminum and very low in phosphorus availabilities. The soil acidity was neutralized using calcium hidroxide to reduce the original aluminum saturation of 67 to 45,30 and $15 \%$. The quantity of subsoll used per pot was $6 \mathrm{~kg}$ and $60 \mathrm{~kg} / \mathrm{ha}$ of $\mathrm{P}_{2} \mathrm{O}_{3}$ were applied in the form of superphosphate. The varieties of soybeans utilized were Biloxi, Davis and Santa Rosa. The results showed that whichever parameter measured (height of the plant, number of nodes, lenght of internodes, number of trifoliate leaves, dry matter weights of shoots and roots) there were no significant differences within each variety for the concentration of aluminum saturation, showing that the three varieties were tolerant to Al. These results were confirmed with two wheat varieties BH-1146 (tolerant to Al) and Siete Cermos (susceptible) which were sown in the same pots. The variety BH-1146 did no show root lenght differences for the Al saturation treaments confirming its tolerance. On the other hand the cultivar Siete Cerros showed increase in root lenght as the Al saturation was reduced, showing its suscebility.

Key Words: dry matter, soybean, aluminum saturation, tolerant

\section{INTRODUÇÃO}

Desde 1970, a produção e o consumo de soja quadruplicaram no Brasil. Entretanto, sua produtividade agrícola não foi aumentada substancialmente. Incremento na produção de grãos de soja é necessário para atender a demanda mundial de óleo e de proteina dessa leguminosa como fonte alimentar dos homens e dos animais.

Um dos fatores que limitam a produtividade da soja é a acidez do solo. A maioria dos solos tropicais sob vegetação de cerrado onde esta leguminosa tem sua maior fronteira de expansão no Brasil, tem perfil ácido, e consequentemente, teor de alumínio (Al) na solução e na forma trocável em niveis tóxicos para as plantas. Altos rendimentos agrícolas ne- cessitam substratos que permitam o desenvolvimento das raízes sem obstáculos físicos e químicos. A pesquisa tem demonstrado que o desenvolvimento do sistema radicular pode ser severamente limitado devido à presença de quantidades apreciáveis de $\mathrm{Al}$ em solos ácidos. $O$ calcário é comumente usado para neutralizar a acidez do solo. Infelizmente, a sua ação corretiva não ultrapassa as camadas superficiais do solo, impossibilitando o crescimento normal das raizes das plantas que necessitam de maior volume do solo a explorar, inclusive em profundidade. A consequância da presença de $\mathrm{Al}$ em nível tóxico no subsolo, é o mau desenvolvimento das raizes. Infere-se que para uma alta produção é necessário o desenvolvimento de raizes nas camadas mais profundas para a absorção de água e de nutrientes.

\footnotetext{
${ }^{2}$ Bolsista de CNPq.

Sci. agric., Piracicaba, 52(2):257-262, mai./ago. 1995
} 
Com a tecnologia atualmente existente, não há método prático e econômico para incorporar o calcário mais profundamente. Uma alternativa, seria desenvolver cultivares tolerantes ao Al. A tolerância das plantas à acidez do solo está ligada à capacidade de absorção de nutrientes e à sensibilidade à toxidez, caracteristicas que dependem da variação genética das espécies e mesmo de cultivares dentro de uma mesma espécie (FOY \& BROWN, 1964). Um trabalho pioneiro de comportamento da soja à acidez do solo foi feito por ARMINGER et al. (1968), que testando 48 cultivares, mostraram diferenças em tolerância ao $\mathrm{Al}$ em subsolo ácido de Bladen. Entre os cultivares promissores destacaramse Biloxi, Perry e Mandarin. Em estudos a campo, MUZILLI et al. (1978) estimaram entre os dez cultivares de soja avaliados sob saturação de $\mathrm{Al}$ (10 a $25 \%$ ), que os cvs. UFV-1, Viçoja e Bossier foram tolerantes, enquanto Santa Rosa, Santa Ana e Hutton apresentaram comportamento intermediário e o restante, foi sensível ou muito sensível ao Al.

$O$ objetivo deste trabalho foi verificar o comportamento $\mathrm{em}$ casa de vegetação, de cultivares de soja e de trigo às saturações de $\mathrm{Al}$ de um subsolo ácido.

\section{MATERIAL E METODOS}

$O$ experimento foi conduzido em casa de vegetação da Seção de Leguminosas do IAC. A amostra de solo utilizado e acondicionado em vasos foi um subsolo (40-60 cm) do município de Morro Agudo (SP), classificado como Latossolo Vermelho Amarelo fase rasa. A análise pela metodologia de CATANI et al. (1955), revelou as seguintes características químicas: $\mathrm{pH} 4,4$; matéria orgânica $0,7 \%$; Ca $2,4 \mathrm{meq} / 100 \mathrm{~g}$; $\mathrm{Mg} 1,6 \mathrm{meq} / 100 \mathrm{~g}$; $\mathrm{K} 0,46 \mathrm{meq} /$ $100 \mathrm{~g} ; \mathrm{PO}_{4}^{-3} 0,02 \mathrm{meq} / 100 \mathrm{~g} ; \mathrm{Al} 9,2 \mathrm{meq} / 100 \mathrm{~g}$ e saturação de Al $67 \%$. A acidez do substrato foi neutraljzado incubando-se com doses de hidróxido de cálcio, no intuito de reduzir a saturação de $\mathrm{Al}$ originalmente de $67 \%$ para 45,30 , e $15 \%$, sendo utilizados $6 \mathrm{~kg}$ de solo/vaso. Na semeadura da soja foram aplicados o equivalente a $60 \mathrm{~kg} / \mathrm{ha} \mathrm{de} \mathrm{P}_{2} \mathrm{O}_{5}$ na forma de superfosfato simples. Foram cultivados inicialmente os cvs. de soja Biloxi, Davis e Santa Rosa. Seis sementes de soja inoculadas com Bradyrhizobium foram postas a germinar por vaso. Dez dias após a germinação, foi feito o desbaste deixando-se três plantas/vaso. $O$ delineamento estatístico utilizado foi o inteiramente ao acaso em esquema fatorial (indices de saturação de $\mathrm{Al} \times$ cultivares), com três repeti̧̧óes. Quarenta dias após a germinação, foram efetuadas as seguintes anotaçס̌es: altura das plan- tas, número de folhas trifoliadas, número de nós e internódios; e o corte das plantas rente ao solo para determinar-se a produção de matéria seca da parte aérea e da raiz. Somente os tecidos da parte aérea foram analisados (BATAGLIA et al. 1983) quanto à concentraçăo de nutrientes. Posteriormente, no mesmo substrato, foi cultivado trigo 'BH-1146' e 'Siete Cerros', até 30 dias, quando foi determinado o comprimento do seu sistema radicular. Para a discussão do efeito dos tratamentos, os dados coletados e determinados foram submetidos à análise de variância.

\section{RESULTADOS E DISCUSSĀO}

\section{Soja}

Houve sintomas de colapso do peciolo que se caracterizaram por dobramento do pecíolo, folhas pouco desenvolvidas e queda das mesmas, em algumas plantas dos três cultivares submetidas a saturação de Al de $67 \%$, conforme podem ser observados na Figura 1. Sintomas semelhantes foram relatados por ARMINGER et al. (1968) que consideraram-nos como secundários de toxidez de Al ou de deficiência de Ca, e por MASCARENHAS et al. (1992) que atribuíram à deficiência de $\mathrm{Ca}$ provocado pelo menor fluxo de massa ocorrido num curto periodo de crescimento da soja.

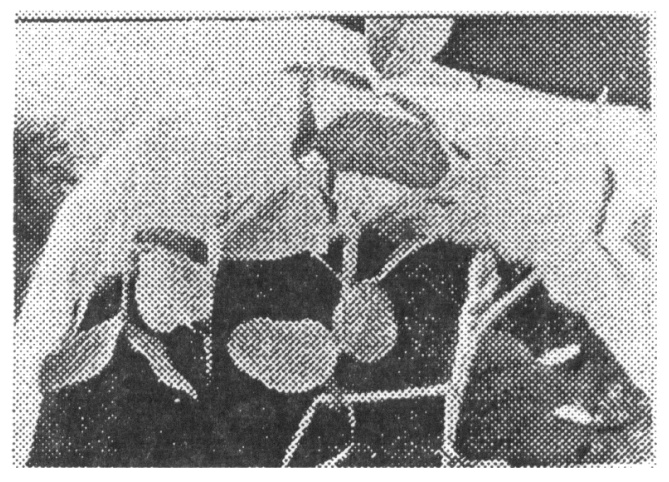

Figura 1. Colapso do peciolo em algumas plantas de soja nos três cultivares de soja à $67 \%$ de saturação de alumínio.

Os índices de saturação de Al não afetaram a altura das plantas, número de folíolos trifoliados, número de nós e tamanho de internódios dentro de cada cultivar, conforme apresentados na TABELA 1. Os genótipos testados são muito sensiveis ao fotoperiodo (origem norte americana de alta latitude). Esse fator induz as plantas ao florescimento em 
TABELA 1. Altura de planta, número de folhas trifoliadas, número de nós e comprimento de internódios

\begin{tabular}{|c|c|c|c|c|}
\hline $\begin{array}{c}\text { Saturação } \\
\text { de } \\
\text { Al }\end{array}$ & $\begin{array}{c}\text { Altura } \\
\text { da } \\
\text { planta }\end{array}$ & $\begin{array}{l}\mathrm{N}^{\circ} \text { de } \\
\text { folhas } \\
\text { trifoliadas }\end{array}$ & $\begin{array}{c}\mathrm{N}^{\circ} \\
\text { de } \\
\text { nós }\end{array}$ & $\begin{array}{l}\text { Comprimento de } \\
\text { internódios }\end{array}$ \\
\hline$\%$ & $\mathrm{~cm}$ & \multicolumn{2}{|c|}{ Biloxi } & $\mathrm{cm}$ \\
\hline 67 & 32,1 & 5 & 6 & 5,8 \\
\hline 45 & 30,6 & 4 & 6 & 5,3 \\
\hline 30 & 34,6 & 4 & 6 & 6,1 \\
\hline 15 & 32,3 & 4 & 6 & 5,9 \\
\hline Média & 32,4 & 4,3 & 6 & 5,8 \\
\hline \multicolumn{5}{|c|}{ Davis } \\
\hline 67 & 24,3 & 5 & 6 & 4,0 \\
\hline 45 & 21,6 & 4 & 6 & 3,9 \\
\hline 30 & 25,4 & 5 & 6 & 4,2 \\
\hline 15 & 23,8 & 5 & 6 & 4,0 \\
\hline Média & 23,8 & 4,8 & 6 & 4,0 \\
\hline \multicolumn{5}{|c|}{ Santa Rosa } \\
\hline 67 & 27,4 & 5 & 6 & 4,6 \\
\hline 45 & 26,3 & 5 & 6 & 4,4 \\
\hline 30 & 27,6 & 5 & 6 & 47 \\
\hline 15 & 29,9 & 5 & 6 & 4,7 \\
\hline Média & 27,8 & 5 & 6 & 4,6 \\
\hline
\end{tabular}

baixas latitudes, (Hinson, 1969), mesmo que não tenham apresentado crescimento em altura, no periodo em que foi conduzida a experimentação. As plantas do cultivar Biloxi apresentaram as maiores alturas e internódios mais longos, enquanto as da Santa Rosa mostraram pequena diferença nessas variáveis; as plantas do cultivar Davis, por outro lado, mostraram ser as mais baixas, com internódios mais curtos, característica de um genótipo precoce.
Apesar de as plantas do cultivar Biloxi terem apresentado maior altura, produziram menos matéria seca do que as dos demais (TABELA 2). Isso se deve a sua característica genética de apresentar maior crescimento dos internódios, sem uma correspondente produção ou acúmulo de matéria seca. A análise de variância mostrou que não houve diferenças entre tratamentos. Ao se desdobrar os graus de liberdade dos tratamentos, o teste $\mathrm{F}$ mostrou somen-

TABELA 2. Média de produção de matéria seca de parte aérea e das raízes dos três cultivares.

\begin{tabular}{|c|c|c|c|c|c|c|}
\hline \multirow{2}{*}{$\begin{array}{c}\text { Saturação } \\
\text { de } \\
\text { alumínio }\end{array}$} & \multicolumn{3}{|c|}{ Parte aérea } & \multicolumn{3}{|c|}{ Raizes } \\
\hline & Biloxi & Davis & Sta Rosa & Biloxi & Davis & Sta Rosa \\
\hline$\%$ & -- & --- & -- & -- & -- & -- \\
\hline 67 & 2,7 & 2,7 & 3,6 & 1,2 & 1,3 & 1,3 \\
\hline 45 & 2,9 & 3,2 & 3,7 & 1,2 & 1,2 & 1,3 \\
\hline 30 & 3,3 & 3,8 & 4,2 & 1,3 & 1,4 & 1,4 \\
\hline 15 & 3,0 & 3,0 & 4,4 & 1,3 & 1,3 & 1,4 \\
\hline Média & $2,98 \mathrm{~B}$ & $3,18 \mathrm{~B}$ & $3,98 \mathrm{~A}$ & 1,25 & 1,30 & 1,35 \\
\hline
\end{tabular}

Médias seguidas da mesma de letra, não apresentam diferenças significativas ao nível de $5 \%$, pelo teste Duncan. 
te diferenças significativas entre os cultivares, sendo o 'Santa Rosa' superior aos outros dois, que por sua vez, foram semelhantes entre si, conforme as produçães médias de matéria seca. Dados semelhantes foram obtidos por SARTAIN \& KAMPRATH (1978).

Pela TABELA 2, observaram-se que não houve diferenças significativas na produção de matéria seca das raizes a níveis de saturação de $\mathrm{Al}$ dentro de cada cultivar ou quando comparadas as médias de raizes entre os cultivares. Também não houve diferenças significativas no tamanho de raizes pivotantes dentro de cada cultivar para saturação de Al, (Figura 2,3,4). Este foi também o critério utilizado por SARTAIN \& KAMPRATH (1978) quando trabalharam com soja em solução nutritiva.

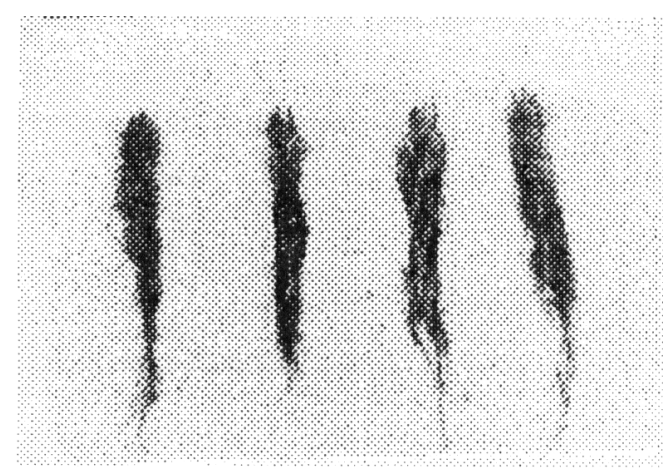

Figura 2. Diferenças no comprimento e no peso da matéria seca das rázes no cultivar Biloxi quando cultivada à $15,30,45$ e $67 \%$ de saturaçăo de alumínio.

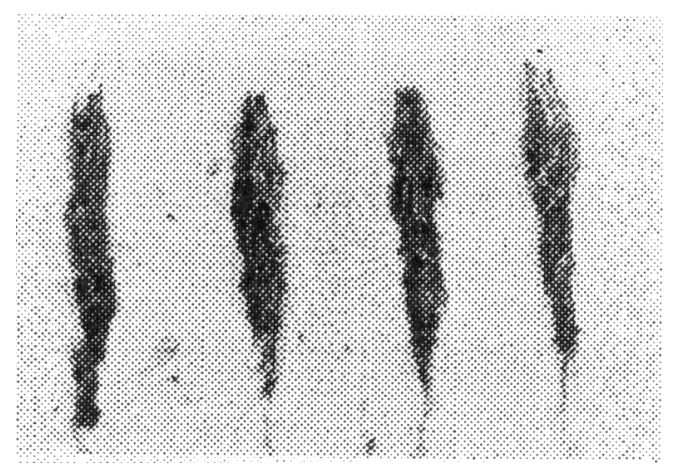

Figura 3. Diferenças no comprimento e no peso da matéria seca das raizes no cultivar Davis quando cultivada d̀ $15,30,45$ e $67 \%$ de saturação de alumínio.

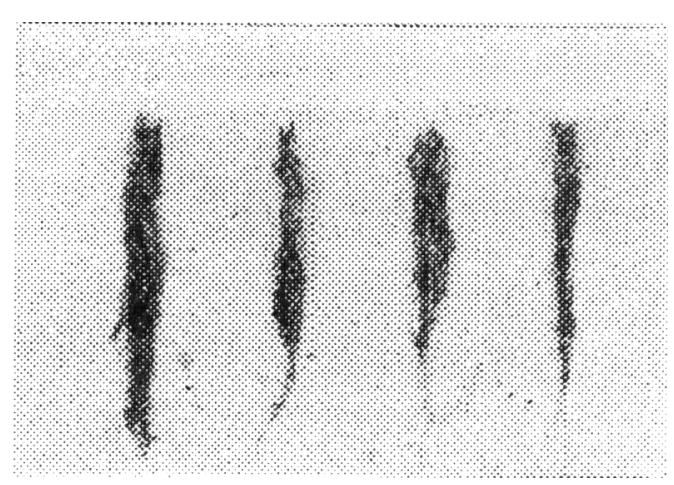

Figura 4. Diferenças no comprimento e no peso da matéria seca das raizes no cultivar Santa Rosa quando cultivada à $15,30,45$ e $67 \%$ de saturação de alumínio.

A TABELA 3 refere-se às médias dos teores de macro e micronutrientes de parte aérea. Notou-se que as plantas do cultivar Santa Rosa apresentaram o maior decréscimo no teor de $\mathrm{Al}$ com a diminuição da saturação do elemento, atingindo $77,3 \%$ do máximo, seguido das plantas do 'Biloxi' $(85,0 \%)$ e das do 'Davis' $(90,1 \%)$. À medida que foi diminuída a saturação de $\mathrm{Al}$, houve um aumento nos teores de $\mathrm{Ca}$ em todos os cultivares. Os teores de $\mathrm{Mg}$ não se modificaram, independentemente da saturação de $\mathrm{Al}$, indicando que o cálcio talvez não interfira na atividade do $\mathrm{Mg}$.

\section{Trigo}

Do cultivo do trigo, os dados das raizes das plantas são mostrados na TABELA 4. O cultivar de trigo $\mathrm{BH}-1146$ revelou ser tolerante e não houve diferenças no tamanho das raizes nas saturações de $\mathrm{Al}$ estudadas. Por outro lado, o comportamento do cultivar Siete Cerros evidenciou que à medida que se reduziu a saturação de $\mathrm{Al}$, houve crescimento significativo das raizes (TABELA 4 e Figuras $5,6,7,8$ ), o que confirmam que os valores das saturaç⿸es de Al estavam adequadas para o presente estudo e que os cultivares de soja Biloxi, Davis e Santa Rosa são altamente tolerantes ao $\mathrm{Al}$, confirmando dados de ARMINGER et al. (1968) para 'Biloxi', e MUZILLI et al. (1978) para os cvs. Santa Rosa e Davis. Este último sendo mostrado pela primeira vez como tolerante ao Al. É importante salientar que os cultivares Biloxi, Santa Rosa e Davis foram também tolerantes ao Mn , em trabalhos de (MIRANDA et al, 1982; MASCARENHAS et al, 1982; MASCARENHAS et al, 1990). 
TABELA 3. Teores de macro e micronutrientes na parte aérea das plantas dos cultivares dee soja.

\begin{tabular}{|c|c|c|c|c|c|c|c|c|}
\hline $\begin{array}{l}\text { Saturação } \\
\text { de Al }\end{array}$ & $\mathbf{N}$ & $\mathbf{P}$ & $\mathrm{K}$ & $\mathrm{Ca}$ & $\mathrm{Mg}$ & $\mathrm{Al}$ & $\mathrm{Fe}$ & $\mathbf{M n}$ \\
\hline$\%$ & \multicolumn{5}{|c|}{$-----\frac{{ }_{\text {Biloxi }}}{\underline{1}}----$} & \multicolumn{3}{|c|}{$--\mathrm{ppm}--$} \\
\hline 67 & 2,93 & 0,119 & 2,80 & 0,39 & 0,54 & 314 & 219 & 84 \\
\hline 45 & 3,15 & 0,110 & 2,64 & 0,46 & 0,51 & 267 & 184 & 76 \\
\hline 30 & 2,86 & 0,116 & 2,67 & 0,53 & 0,50 & 278 & 223 & 92 \\
\hline \multirow[t]{2}{*}{15} & 2,86 & 0,113 & 2,82 & 0,60 & 0,52 & 284 & 224 & 90 \\
\hline & \multicolumn{5}{|c|}{ Davis } & & & \\
\hline 67 & 3,03 & 0,124 & 2,74 & 0,42 & 0,54 & 374 & 161 & 92 \\
\hline 45 & 3,01 & 0,119 & 2,79 & 0,49 & 0,50 & 337 & 152 & 94 \\
\hline 30 & 2,93 & 0,114 & 2,80 & 0,56 & 0,54 & 340 & 197 & 98 \\
\hline \multirow[t]{2}{*}{15} & 2,79 & 0,125 & 2,63 & 0,59 & 0,55 & 338 & 194 & 86 \\
\hline & \multicolumn{5}{|c|}{ Santa Rosa } & & & \\
\hline 67 & 3,45 & 0,121 & 2,60 & 0,31 & 0,52 & 370 & 218 & 86 \\
\hline 45 & 3,37 & 0,120 & 3,12 & 0,52 & 0,58 & 331 & 223 & 79 \\
\hline 30 & 3,37 & 0,148 & 3,06 & 0,56 & 0,61 & 304 & 205 & 82 \\
\hline 15 & 3,45 & 0,124 & 3,06 & 0,60 & 0,59 & 286 & 205 & 84 \\
\hline
\end{tabular}

TABELA 4. Comprimento de raizes dos cultivares de trigo BH-1146 (tolerante) e Siete Cerros (susceptivel)

\begin{tabular}{ccc}
\hline \hline Saturação de Al & BR-1146 & Siete Cerros \\
\hline$\%$ & $--\frac{1}{15,03 \mathrm{a}}$ & $\mathrm{cm}---\frac{1}{1,25 \mathrm{c}}-$ \\
67 & $17,37 \mathrm{a}$ & $5,36 \mathrm{~b}$ \\
45 & $17,96 \mathrm{a}$ & $8,73 \mathrm{a}$ \\
30 & $15,72 \mathrm{a}$ & $10,03 \mathrm{a}$ \\
15 & $16,52 \mathrm{~A}$ & $6,34 \mathrm{~B}$ \\
Média & & \\
\hline \hline
\end{tabular}

Médias seguidas da mesma de letra, não apresentam diferenças significativas ao nível de $5 \%$, pelo teste Duncan.

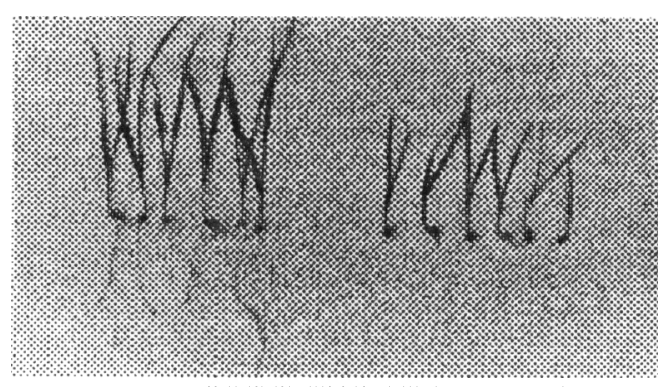

Figura 5. Plantas do cultivar de trigo Siete Cerros (susceptível ao $\mathrm{Al}$ ) sem raizes a $67 \%$ de saturação de $\mathrm{Al}$, e do 'BH-1146' (tolerante), com raizes.

Sci. agric., Piracicaba, 52(2):257-262, mai./ago. 1995

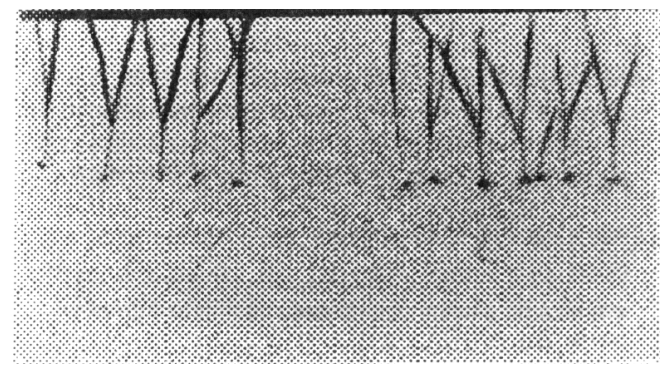

Figura 6. Plantas do cultivar de trigo Siete Cerros (susceptivel ao Al) com pouco desenvolvimento de raizes a $45 \%$ de saturação de Al, e do 'BH-1146' (tolerante), com desenvolvimento normal de raizes. 


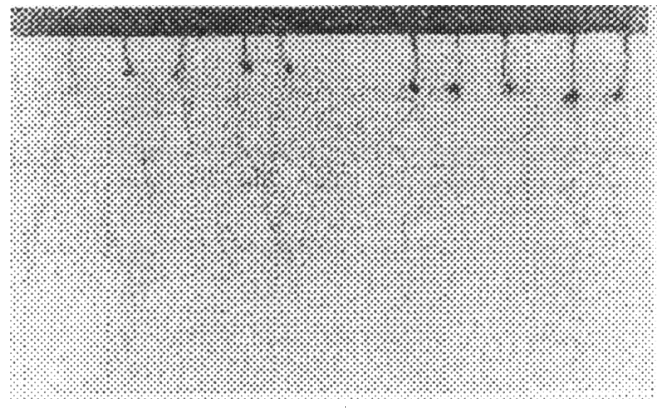

Figura 7. Plantas do cultivar de trigo Siete Cerros (susceptível ao $\mathrm{Al}$ ) com melhor desenvolvimento de raizes a $30 \%$ de saturação de $\mathrm{Al}$, e do 'BH-1146' (tolerante), com desenvolvimento normal de raizes.

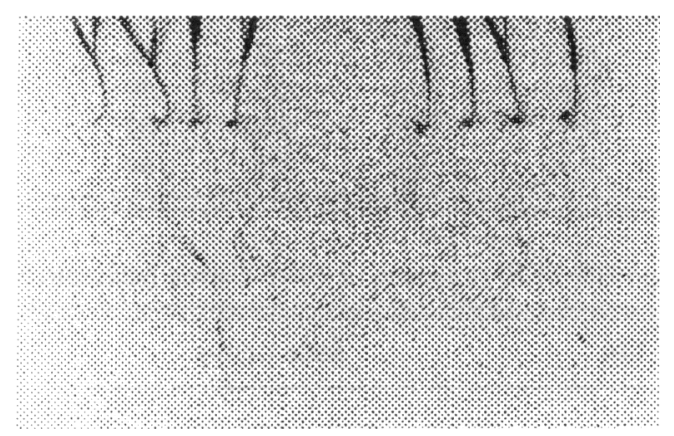

Figura 8. Plantas do cultivar de trigo Siete Cerros (susceptível ao $\mathrm{Al}$ ) raizes bem desenvolvidos a $15 \%$ de saturação de $\mathrm{Al}$, e do ' $\mathrm{BH}$ 1146 ' (tolerante), com desenvolvimento normal de raízes.

\section{REFERENCIAS BIBLIOGRÁFICAS}

ARMINGER, W.H.; FOY, C.D.; FLEMING, A.L. \& CALDWELL, B.E. Differential tolerance of soybean varieties to an acid soil high in exchangeable aluminum. Agronomy Journal, Madison, v.60, p.67-70, 1968.

BATAGLIA, O.C.; TEIXEIRA, J.P.F.; FURLANI, P.R.; FURLANI, A.M.C.; GALLO, J.R. Métodos de análise química de plantas. Campinas, Instituto Agronômico, 1978, 31 p. (Circular, 87).
CATANI, R.A.; GALLO, J.R.; GARGANTINI, H. Amostragem de solo, métodos de análises, interpretação e indicações gerais para fins de fertilidade. Campinas, Instituto Agronômico, 1955. 29p. (Boletim 69).

FOY, C.D.; BROWN, I.C. Toxic factors in acid soils: II. Differential aluminum tolerance of plant species. Soll Science of Society Proceedings, v.28, n. 1, p.27-32, 1964.

HINSON, K. Soybeans in Florida. Agricultural Experimental Stations, University of Florida, Gainesville, 1969, Bulletin 716, 121p.

MASCARENHAS, H.A.A; MIRANDA, M.A.C.; RAMOS, L.C.B; FURLANI, P.R.; BATAGLIA, O.C. Comportamento de três cultivares de soja em função do manganês do solo. Bragantia, Campinas, v.41, p.225-230, 1982.

MASCARENHAS, H.A.A; MIRANDA, M.A.C.; TANAKA, R.T.; FALIVENE, S.M.P.; DECHEN, A.R. Comportamento de cultivares precoces de soja em solução nutritiva contendo diferentes níveis de manganês. Pesquisa Agnopecuária Brasileira, Brasilia, v 25, n 4, p.609-615, 1990.

MASCARENHAS, H.AA; MIRANDA, M.AC.; TANAKA, R.T. Colapso do peciolo em folhas de soja. Pesquisa Agropecuária Brasilleira, Brasilia, v.27, n.2, p.343-348, fevereiro, 1992.

MIRANDA, M.A.C.; MASCARENHAS, H.A.A.; BULISANI, E.A.; VALADARES, J.M.A.S.; HIROCE, R. Comportamento de dois cuhivares de soja em função do manganês no solo. Bragantia, Campinas, v.41, p.135-143, 1982.

MUZILLI, O.; SANTOS, D.; PALHANO, J.B.; MANETTI $F^{\circ}$, J.; LANTMANN, A.F;; GARCIA, A.; CATENO, 4 Tolerância de cultivares de soja e do trigo a acidè ito solo. Revista Brasileira de Ciência do Solo, Campmas v.2, p.34-40, 1978.

SARTAIN, J.B.; KAMPRATH, E.J. Aluminum tolerance of soybean cultivars based on root elongation in solution culture compared with growth in acid soil. Agronomy Journal, Madison, v.70, p.17-20, 1978.

$\overline{\text { Recebido para publicação em } 09.01 .95}$

Aceito para publicação em 26.04 .95 